\title{
A generalized periodic boundary value problem for the one-dimensional $p$-Laplacian
}

\author{
by DAQING JiAng and JUNYU WANG (Changchun)
}

\begin{abstract}
The generalized periodic boundary value problem $-\left[g\left(u^{\prime}\right)\right]^{\prime}=f\left(t, u, u^{\prime}\right)$, $a<t<b$, with $u(a)=\xi u(b)+c$ and $u^{\prime}(b)=\eta u^{\prime}(a)$ is studied by using the generalized method of upper and lower solutions, where $\xi, \eta \geq 0, a, b, c$ are given real numbers, $g(s)=|s|^{p-2} s, p>1$, and $f$ is a Carathéodory function satisfying a Nagumo condition. The problem has a solution if and only if there exists a lower solution $\alpha$ and an upper solution $\beta$ with $\alpha(t) \leq \beta(t)$ for $a \leq t \leq b$.
\end{abstract}

1. Introduction. The present paper is a continuation of the papers [1] and $[2]$.

In this paper, we study the following generalized periodic boundary value problem for the one-dimensional $p$-Laplacian:

$$
\left\{\begin{array}{l}
-\left[g\left(u^{\prime}\right)\right]^{\prime}=f\left(t, u, u^{\prime}\right), \quad t \in I:=[a, b] \\
u(a)=\xi u(b)+c, \quad u^{\prime}(b)=\eta u^{\prime}(a)
\end{array}\right.
$$

by using the generalized method of upper and lower solutions. Here $\xi, \eta \geq 0$, $a, b, c$ are given real numbers, $g(s)=|s|^{p-2} s, p>1$, and $f(t, u, v)$ is a Carathéodory function satisfying a Nagumo condition.

We name the problem a generalized periodic boundary value problem since the periodic boundary value problem is its particular case.

We call a function $\alpha: I \rightarrow \mathbb{R}$ a lower solution to problem (1.1) if $\alpha \in$ $C^{1}(I), g\left(\alpha^{\prime}\right) \in A C(I)$, and

$$
\left\{\begin{array}{l}
-\left[g\left(\alpha^{\prime}(t)\right)\right]^{\prime} \leq f\left(t, \alpha(t), \alpha^{\prime}(t)\right) \quad \text { for a.e. } t \in I, \\
\alpha(a)=\xi \alpha(b)+c, \quad \alpha^{\prime}(b) \leq \eta \alpha^{\prime}(a),
\end{array}\right.
$$

where $A C(I)$ is the set of all absolutely continuous functions defined on $I$.

1991 Mathematics Subject Classification: 34B15, 34B10.

Key words and phrases: generalized periodic boundary value problem, $p$-Laplacian, upper and lower solutions, Carathéodory function, Nagumo condition.

The second author is supported by NNSF of China. 
Similarly, a function $\beta: I \rightarrow \mathbb{R}$ is called an upper solution to (1.1) if $\beta \in C^{1}(I), g\left(\beta^{\prime}\right) \in A C(I)$, and

$$
\left\{\begin{array}{l}
-\left[g\left(\beta^{\prime}(t)\right)\right]^{\prime} \geq f\left(t, \beta(t), \beta^{\prime}(t)\right) \quad \text { for a.e. } t \in I, \\
\beta(a)=\xi \beta(b)+c, \quad \beta^{\prime}(b) \geq \eta \beta^{\prime}(a) .
\end{array}\right.
$$

A function $u: I \rightarrow \mathbb{R}$ is said to be a solution to (1.1) if it is both a lower solution and an upper solution to (1.1).

We call a function $f: I \times \mathbb{R}^{2} \rightarrow \mathbb{R}$ a Carathéodory function if the following two conditions are satisfied:

(1) for almost all $t \in I$, the function $(u, v) \rightarrow f(t, u, v)$ is continuous on $\mathbb{R}^{2}$, and

(2) for every $(u, v) \in \mathbb{R}^{2}$, the function $t \rightarrow f(t, u, v)$ is measurable on $I$.

The function $f$ is said to satisfy a Nagumo condition on the set

$$
D:=\{(t, u, v): t \in I, \alpha(t) \leq u \leq \beta(t), v \in \mathbb{R}\}
$$

for given $\alpha, \beta \in C(I)$ with $\alpha(t) \leq \beta(t)$ on $I$ if there exists a positive measurable function $k \in L_{\sigma}(I), 1 \leq \sigma \leq \infty$, and a positive continuous function $H \in C\left(\mathbb{R}_{+}\right), \mathbb{R}_{+}:=[0, \infty)$ such that

$$
|f(t, u, v)| \leq k(t) H(|v|) \quad \text { a.e. on } D
$$

and

$$
\int_{g(A)}^{\infty} \frac{|G(s)|^{(\sigma-1) / \sigma}}{H(|G(s)|)} d s>B^{(\sigma-1) / \sigma}\|k\|_{\sigma},
$$

where $G$ is the function inverse to $g$,

$$
\begin{aligned}
& A:=\max \{|\beta(a)-\alpha(b)|,|\beta(b)-\alpha(a)|\} /(b-a), \\
& B:=\max \{\beta(t): t \in I\}-\min \{\alpha(t): t \in I\}
\end{aligned}
$$

and

$$
\|k\|_{\sigma}:= \begin{cases}\left(\int_{a}^{b}|k(s)|^{\sigma} d s\right)^{1 / \sigma} & \text { if } \sigma \in[1, \infty), \\ \operatorname{ess} \sup \{|k(t)|: t \in I\} & \text { if } \sigma=\infty .\end{cases}
$$

Here we set $B^{0}:=1$ and $|G(s)|^{0}:=1$.

The main result of this paper is as follows.

Theorem 1. Assume that $f$ is a Carathéodory function satisfying a Nagumo condition. Then a necessary and sufficient condition for the problem (1.1) to have a solution $u$ is that there exists a lower solution $\alpha$ and an upper solution $\beta$ with $\alpha(t) \leq \beta(t)$ on I. Moreover,

$$
\alpha(t) \leq u(t) \leq \beta(t) \quad \text { and } \quad\left|u^{\prime}(t)\right| \leq N \quad \text { on } I,
$$

where $N$ is a constant depending only on $\alpha, \beta, g, H$ and $k$. 
Obviously, Theorem 1 extends and improves Theorem 1 of [1] and Theorem 2.4 of [2].

2. Proof of Theorem 1. The necessity part is obvious. We prove the sufficiency.

Now assume that $\alpha$ and $\beta$ are lower and upper solutions to problem (1.1), respectively, and $\alpha(t) \leq \beta(t)$ on $I$. To prove the existence of solutions (1.1), we consider the modified problem

$$
\left\{\begin{array}{l}
-\left[g\left(u^{\prime}\right)\right]^{\prime}+M g\left(u^{\prime}\right) \\
\quad=f^{*}\left(t, q(t, u), \frac{d}{d t} q(t, u)\right)+M g\left(\frac{d}{d t} q(t, u)\right), \quad t \in I, \\
u(a)=\xi q(b, u(b))+c, \quad u^{\prime}(b)=\eta u^{\prime}(a),
\end{array}\right.
$$

where $M$ is a positive number such that $e^{M(b-a)}>\eta^{p-1}$,

$$
q(t, u):= \begin{cases}\alpha(t) & \text { if } u<\alpha(t), t \in I \\ u & \text { if } \alpha(t) \leq u \leq \beta(t), t \in I, \\ \beta(t) & \text { if } u>\beta(t), t \in I\end{cases}
$$

and

$$
f^{*}(t, u, v):= \begin{cases}f(t, u,-N) & \text { if } v<-N \\ f(t, u, v) & \text { if }|v| \leq N \\ f(t, u, N) & \text { if } v>N\end{cases}
$$

Here we choose $N$ so large that

$$
N>\max \left\{\left|\alpha^{\prime}(t)\right|,\left|\beta^{\prime}(t)\right|: t \in I\right\}+A
$$

and

$$
\int_{g(A)}^{g(N)} \frac{|G(s)|^{(\sigma-1) / \sigma}}{H(|G(s)|)} d s>B^{(\sigma-1) / \sigma}\|k\|_{\sigma}
$$

( $A$ and $B$ are defined by (1.4) and (1.5) respectively). (1.3) assures the existence of such an $N$.

Lemma 1. For any $u \in E:=C^{1}(I)$, the following two statements hold:

(1) $(d / d t) q(t, u(t))$ exists for a.e. $t \in I$.

(2) If $u_{0}, u_{j} \in E$ and $u_{j} \rightarrow u_{0}$ in $E$, then

$$
\frac{d}{d t} q\left(t, u_{j}(t)\right) \rightarrow \frac{d}{d t} q\left(t, u_{0}(t)\right) \quad \text { for a.e. } t \in I .
$$

Proof. The proof can be found in $[1,3]$.

Lemma 2. Let $u$ be a solution to (2.1). Then

(1) $\alpha(t) \leq u(t) \leq \beta(t)$ on $I$, and

(2) $\left|u^{\prime}(t)\right| \leq N$ for all $t \in I$.

That is to say, the solution $u$ is also a solution to (1.1). 
Proof. We first prove that $u(t) \leq \beta(t)$ on $I$. Let $y(t)=u(t)-\beta(t)$. Then we have

$$
y(a)=\xi[q(b, u(b))-\beta(b)] \leq 0, \quad y^{\prime}(b) \leq \eta y^{\prime}(a) .
$$

Assume now that $y(t)>0$ for some $t \in(a, b]$. Then there exists a point $t^{*} \in(a, b]$ such that $y\left(t^{*}\right)$ is the positive maximum value. We can distinguish two cases.

Case (i): $t^{*}<b$. In this case, $y^{\prime}\left(t^{*}\right)=u^{\prime}\left(t^{*}\right)-\beta\left(t^{*}\right)=0$ and there exists a point $t_{1} \in\left[a, t^{*}\right)$ such that $y\left(t_{1}\right)=0$ and $y(t)>0$ in $\left(t_{1}, t^{*}\right]$. Thus, we have

$$
\begin{aligned}
-\left[g\left(\beta^{\prime}(t)\right)\right]+M g\left(\beta^{\prime}(t)\right) & \geq f\left(t, \beta(t), \beta^{\prime}(t)\right)+M g\left(\beta^{\prime}(t)\right) \\
& =-\left[g\left(u^{\prime}(t)\right)\right]^{\prime}+M g\left(u^{\prime}(t)\right) \quad \text { a.e. on }\left[t_{1}, t^{*}\right]
\end{aligned}
$$

(since $q(t, u(t))=\beta(t)$ and $\left|\beta^{\prime}(t)\right| \leq N$ on $\left[t_{1}, t^{*}\right]$ ), i.e.,

$$
\frac{d}{d t}\left\{e^{-M t}\left[g\left(u^{\prime}(t)\right)-g\left(\beta^{\prime}(t)\right)\right]\right\} \geq 0 \quad \text { a.e. on }\left[t_{1}, t^{*}\right] .
$$

This shows that

$$
e^{-M t}\left[g\left(u^{\prime}(t)\right)-g\left(\beta^{\prime}(t)\right)\right] \leq e^{-M t^{*}}\left[g\left(u^{\prime}\left(t^{*}\right)\right)-g\left(\beta^{\prime}\left(t^{*}\right)\right)\right]=0 \quad \text { on }\left[t_{1}, t^{*}\right],
$$

i.e., $y^{\prime}(t) \leq 0$ on $\left[t_{1}, t^{*}\right]$. Consequently, we get a contradiction: $0=y\left(t_{1}\right) \geq$ $y\left(t^{*}\right)>0$.

Case (ii): $t^{*}=b$. If $y^{\prime}(b)=0$, we can get a contradiction again as in Case (i). If $y^{\prime}(b)>0$, then by $(2.2)$,

$$
y(a)=0, \quad \eta>0 \quad \text { and } \quad y^{\prime}(a)>0 .
$$

When $y(t)>0$ in $(a, b]$, we easily obtain

$$
\frac{d}{d t}\left\{e^{-M t}\left[g\left(u^{\prime}(t)\right)-g\left(\beta^{\prime}(t)\right)\right]\right\} \geq 0 \quad \text { a.e. on } I,
$$

as in Case (i), i.e.,

$$
e^{-M b}\left[g\left(u^{\prime}(b)\right)-g\left(\beta^{\prime}(b)\right)\right] \geq e^{-M a}\left[g\left(u^{\prime}(a)\right)-g\left(\beta^{\prime}(a)\right)\right] .
$$

Since $u^{\prime}(b)=\eta u^{\prime}(a)$ and $\beta^{\prime}(b) \geq \eta \beta^{\prime}(a)$, we have

$$
\left(\eta^{p-1}-e^{M(b-a)}\right)\left[g\left(u^{\prime}(a)\right)-g\left(\beta^{\prime}(a)\right)\right] \geq 0,
$$

i.e., $y^{\prime}(a)=u^{\prime}(a)-\beta^{\prime}(a) \leq 0$, which contradicts the assumption $y^{\prime}(a)>0$.

When there is a point $t_{4} \in(a, b)$ such that $y\left(t_{4}\right) \leq 0$, it follows from $(2.3)$ that there exists an interval $\left(t_{2}, t_{3}\right), a \leq t_{2}<t_{3} \leq t_{4}$, such that $y(t)>0$ in $\left(t_{2}, t_{3}\right)$ and $y\left(t_{2}\right)=y\left(t_{3}\right)=0$. Therefore, there is a point $t^{* *} \in\left(t_{2}, t_{3}\right)$ such that $y\left(t^{* *}\right)$ is the positive maximum value of $y(t)$ on $\left[t_{2}, t_{3}\right]$. As in Case (i), we can get a contradiction again. This shows that $u(t) \leq \beta(t)$ on $I$.

In very much the same way, we can prove that $\alpha(t) \leq u(t)$ on $I$. (1) is thus proved. 
The proof of (2) can be found in [1,2]. The Nagumo condition is employed only here.

To prove the existence of solutions to problem (2.1), we define a mapping $\Phi: E \rightarrow E$ by

$$
\begin{aligned}
(\Phi u)(t):= & \int_{a}^{t} G\left(\tau e^{M(r-a)}-\int_{a}^{r} e^{M(r-s)}(F u)(s) d s\right) d r \\
& +\xi q(b, u(b))+c
\end{aligned}
$$

for $u \in E$, where the mapping $F: E \rightarrow L_{\sigma}(I)$ is defined by

$$
\begin{aligned}
(F u)(t):= & f^{*}\left(t, q(t, u(t)), \frac{d}{d t} q(t, u(t))\right) \\
& +M g\left(\frac{d}{d t} q(t, u(t))\right) \quad \forall u \in E
\end{aligned}
$$

and

$$
\tau:=\left[e^{M(b-a)}-\eta^{p-1}\right]^{-1} \int_{a}^{b} e^{M(b-s)}(F u)(s) d s .
$$

Obviously, $F$ is well defined, since for any $u \in E$,

$$
|(F u)(t)| \leq M^{*} k(t)+M g(N) \in L_{\sigma}(I),
$$

where $M^{*}:=\max \{H(s): 0 \leq s \leq N\}$.

LeMma 3. $\Phi$ is a completely continuous mapping.

Pr o o f. Let $w(t)=(\Phi u)(t)$. From the definition of $\Phi$, we have for $u \in E$,

$$
w^{\prime}(t)=G\left(\tau e^{M(t-a)}-\int_{a}^{t} e^{M(t-s)}(F u)(s) d s\right) \in C(I)
$$

and there exists an $N^{*}$, independent of $u$, such that

$$
|\tau|,\left|w^{\prime}(t)\right|,|w(t)| \leq N^{*} \quad(t \in I) .
$$

This shows that $\Phi(E)$ is a bounded subset of $E$.

Since the set

$$
\left\{\tau e^{M(t-a)}-\int_{a}^{t} e^{M(t-s)}(F u)(s) d s: u \in E\right\}
$$

is bounded and equicontinuous on $I$, so is the set $\left\{w^{\prime}(t): u \in E\right\}$. By the Arzelà-Ascoli theorem, $\Phi(E)$ is compact in $E$. 
Let $u_{0}, u_{j} \in E$ and $u_{j} \rightarrow u_{0}$ in $E$. By Lemma 1 and the dominated convergence theorem, we conclude that as $j \rightarrow \infty$,

$$
\begin{aligned}
\tau_{j} & :=\left[e^{M(b-a)}-\eta^{p-1}\right]^{-1} \int_{a}^{b} e^{M(b-s)}\left(F u_{j}\right)(s) d s \\
& \rightarrow\left[e^{M(b-a)}-\eta^{p-1}\right]^{-1} \int_{a}^{b} e^{M(b-s)}\left(F u_{0}\right)(s) d s=: \tau_{0},
\end{aligned}
$$

and hence $\Phi u_{j} \rightarrow \Phi u_{0}$ in $E$. This shows that $\Phi$ is continuous on $E$. The proof is complete.

From Lemma 3, the Schauder fixed point theorem asserts that $\Phi$ has at least one fixed point in $E$. Let $u \in E$ be a fixed point of $\Phi$. Then

$$
\begin{aligned}
u(t)= & \int_{a}^{t} G\left(\tau e^{M(r-a)}-\int_{a}^{r} e^{M(r-s)}\left[f^{*}\left(s, q(s, u(s)), \frac{d}{d s} q(s, u(s))\right)\right.\right. \\
& \left.\left.+M g\left(\frac{d}{d s} q(s, u(s))\right)\right] d s\right) d r+\xi q(b, u(b))+c \quad \text { on } I,
\end{aligned}
$$

where $\tau$ is determined by (2.5). It is easy to see that the fixed point $u$ is a solution to (2.1). Of course, the $u$ is also a solution to (1.1).

Theorem 1 is proved.

\section{References}

[1] W. J. Gao and J. Y. Wang, On a nonlinear second order periodic boundary value problem with Carathéodory functions, Ann. Polon. Math. 62 (1995), 283-291.

[2] J. Y. Wang, W. J. Gao and Z. H. Lin, Boundary value problems for general second order equations and similarity solutions to the Rayleigh problem, Tôhoku Math. J. 47 (1995), 327-344.

[3] M. X. Wang, A. Cabad a and J. J. Nieto, Monotone method for nonlinear second order periodic boundary value problems with Carathéodory functions, Ann. Polon. Math. 58 (1993), 221-235.

Daqing Jiang

Department of Mathematics

Northeast Normal University

Changchun 130024, P.R. China
Junyu Wang

Department of Mathematics

Jilin University

Changchun 130023, P.R. China 